\title{
Experimental and Numerical Investigations of Friction Rings Damping of Blisks
}

\author{
Denis Laxalde ${ }^{* \dagger} ;$ Claude Gibert ${ }^{\dagger}$ and Fabrice Thouverez ${ }^{\dagger}$
}

\begin{abstract}
The use of friction ring dampers for integrally bladed disks (blisks) is investigated numerically and experimentally in this paper. A test rig was developed and consists in an industrial HP compressor blisk rotating inside a vacuum chamber. Excitation is produced through piezoelectric actuators and measured data are obtained from strain gauges. Non-linear resonance curves obtained by stepped sine tests are studied. Interesting phenomena on the behaviour of this damping technology are obtained experimentally. Parametric studies on the influence of the rotation speed or of the excitation level are also presented. A non-linear modal identification method is used in order to extract the modal parameters from the resonance curves. Then a comparison of these experimental results to the results of numerical simulations is proposed. The numerical methods is based on a frequency domain formulation of the system's dynamics; a non-linear modal approach is used. The correlation between the experiments and the predicted results are in quite good agreement given the complexity and the variability of the system and phenomena.
\end{abstract}

\section{Introduction}

Integrally bladed disks (blisks) feature a very weak internal damping and can often pose a risk of HCF. Their need for external damping sources is then crucial to ensure safe operating conditions. The use of friction devices is probably the most popular damping solution in turbomachinery bladed disks applications. However, for blisks in which blade-root joints no longer exists, traditional technologies cannot be used straightforward. A technological answer to this

\footnotetext{
*Address all correspondence to this author.

†École Centrale de Lyon, Laboratoire de Tribologie et Dynamique des Systèmes, 36 avenue Guy de Collongue, 69134 Ecully Cedex, France

${ }^{\ddagger}$ Snecma - Safran group, 77550 Moissy-Cramayel, France
}

issue was recently proposed in [8]; it consists in using friction rings held in contact underside the wheel of the blisk due to centrifugal load. The friction ring dampers can be located anywhere underside the "rim" of the blisk. Figure 1 shows two possible implantations of rings on a blisk. As in traditional fric-

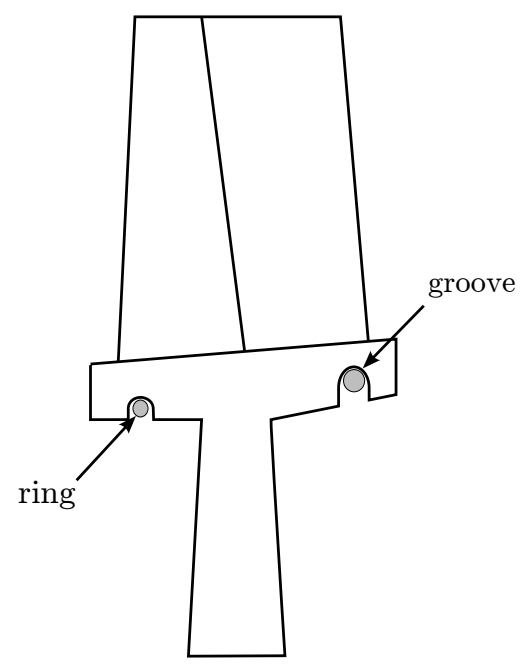

Figure 1. Cross-sectional view of a blisk with possible locations of friction rings

tion devices, damping occurs when relative motion between the disk and the ring takes place.

Friction damping generally involves phenomena of great complexity and variability. Consequently, experimental studies are often needed for better understanding of these phenomena on the one hand and for the validation and adjustment of simulation tools on the other hand. Several studies on friction damping in bladed disks have been presented in the literature. Most of these focus on friction damping in bladed disks assemblies [2, 6, 12, 14]: under-platform dampers, shrouds, blade-root damping,... A structure (often with simplified geometry) is generally isolated in a controlled environment. In rotating conditions, the use of a vacuum chamber is often needed [2, 
$6,12]$ to eliminate the aerodynamic effects. For this study, a dedicated test rig was developed at the Ecole Centrale de Lyon and consists in an industrial HP compressor blisk rotating inside a vacuum chamber.

The paper presents first the methodology and results of this experimental investigation. The excitation device, which consists piezoelectric actuators, is described as well as the measurements system. Nonlinear resonance curves obtained by stepped sine tests are studied. The experimental results show interesting phenomena on the behaviour of this damping technology and several parametric studies are presented such as the influence of the rotation speed or of the excitation force. Then a comparison of these experimental results is proposed with the results of numerical simulations. The numerical methods is based on a frequency domain formulation of the system's dynamics. Both the rings and the blisk finite elements models are presented and several contact models are proposed. The degree of correlation between the experiments and the predicted results is then discussed.

\section{Experimental set-up and methods}

The objectives of the present experimental study are numerous. First, it will provide a better understanding of the friction phenomena which are generally complex all the more so they are part of a whole dynamical system. Second, various parametric studies will be performed in order to evaluate the efficiency of the friction ring damping technology. We are particularly interested in studying the effects of the rotation speed and of the excitation parameters (frequency, shape, amplitude,...). One particularity of the chosen means of excitation is that it is independent of the rotating speed. Though, uncoupling the effects of the two aforementioned parameters (rotation and excitation) may be useful for investigations. Other side effects, such as endurance or wear can be investigated. Finally, the results of these study will be used for the validation and validation and adjustment of numerical simulation tools.

\subsection{The test structure}

This study aims at investigating the damping of friction rings for a blisk. The test structure is a 36 blades HP compressor blisk from a civil engine demonstrator. A view of the mesh of an elementary sector is provided in figure $2 \mathrm{a}$. Three positions for the rings

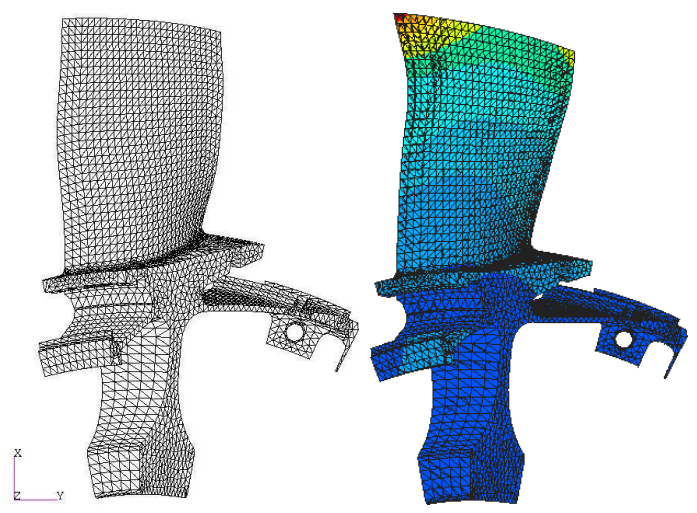

(a)

(b)

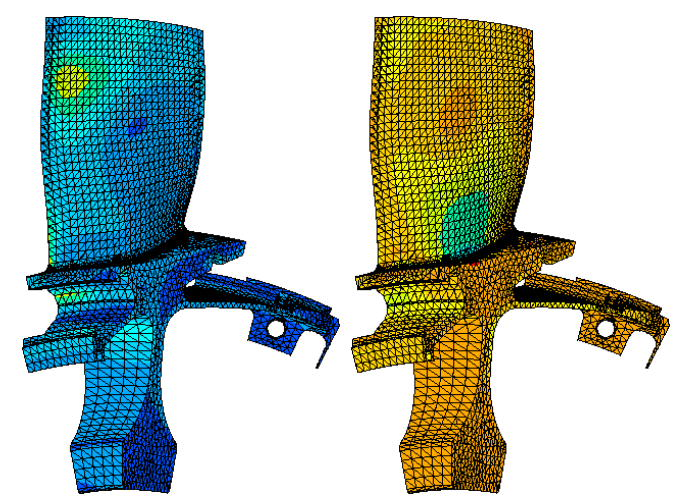

(c)

(d)

Figure 2. The test structure: (a) FE mesh of a sector, (b) mode shape, (c) and (d) strain in the principal directions for the third mode at 4 nodal diameters.

were defined and several rings' dimensions are possible.

The so-called target mode for this study is the third mode at four nodal diameters which deformed shape is shown in figure $2 \mathrm{~b}$ along with strain maps in figures $2 \mathrm{c}$ and $2 \mathrm{~d}$.

\subsection{General description of the test rig}

The blisk presented above is adapted to an existing rig at the "Laboratory of Tribology and Systems Dynamics" at the ECL which is devoted to the study the vibration of rotating parts operating in vacuum conditions to suppress aerodynamic effects (see figure 3 ). This set-up has been initiated by the ADTurb II project [1] and has been used to characterise the effect of friction on the dynamics of rotating blades with 
dovetail or fir tree attachments and featuring cottage roof or under platform dampers $[2,6,17]$. The blisk

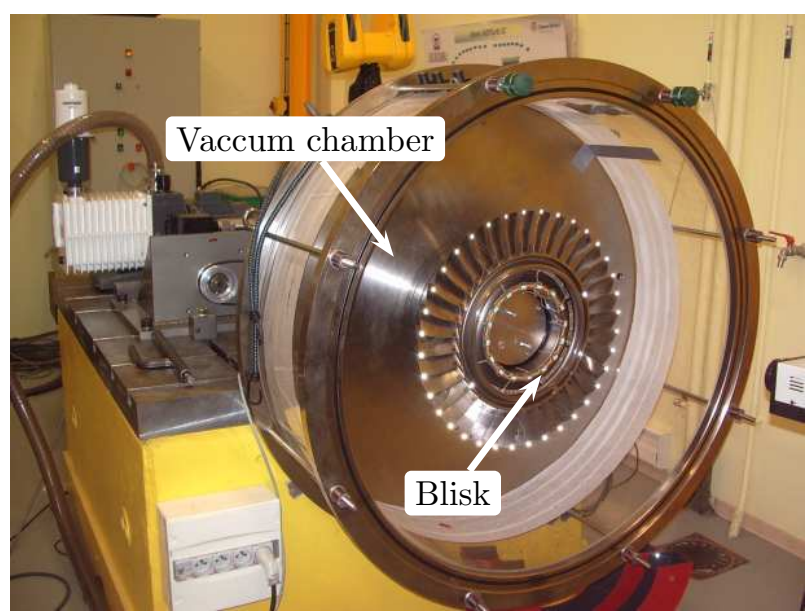

Figure 3. Test rig

was bolted on a very stiff steel supporting disk fitted on the shaft and designed so as to minimise dynamic coupling with the blisk, especially on target modes, while avoiding critical speeds within operating range. The supporting disk enables the attachment of balancing masses, wires and electronic box (see figure 4 . The shaft is driven by an electric motor providing a rotational speed of $0-5000 \mathrm{rpm}$ which is sufficient to obtain significant centrifugal load. Finally, tests are performed at ambient temperature.

\subsection{Excitation}

The excitation is provided by several PZT piezoelectric plates $(30 \times 10 \times 1 \mathrm{~mm})$ working in $d_{31}$ mode and bonded on the blisk by means of insulating and silver conductive epoxy adhesive. 24 piezo-ceramic (PZTs) are positioned circumferentially on the blisk as shown in figures 5 on both the front and rear faces. The choice for these locations was driven by several considerations among:

- high strain is predicted when vibrating on the target modes;

- plain surfaces well suited for bonding of layers in order to maximise their efficiency;

- no perturbation are brought on blades

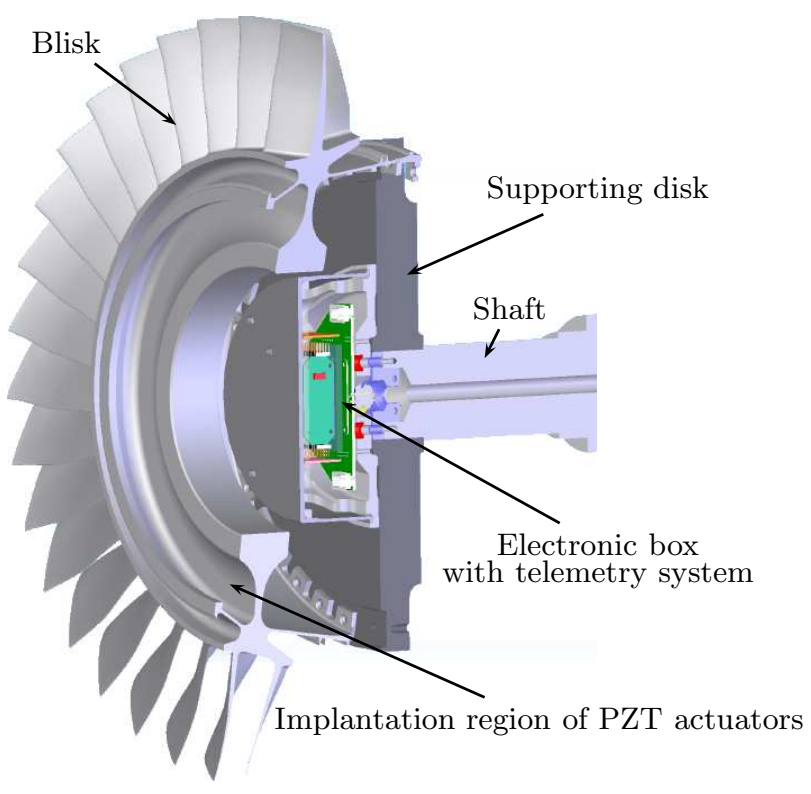

Figure 4. CAD view of the mounting.

- relative easiness of work with (positioning, bonding, wiring...)

These 24 ceramic plates are gathered in 8 groups of 3 PZTs. All ceramics within a given group are connected in parallel with their polarities in the same direction and therefore will transmit stresses to the structure with same phase for a given common voltage. Small and light-weight electronic tension inverters made of DIP-switches assembled on a integrated circuit card are used. These are connected between the input of each group in order to be able to set either 0 or 180 degrees of relative phase and a common voltage between a given group and the common voltage. These are gathered on the front side, bonded on the bottom massive part of the blisk section, allowing the user to access for setting the nodal diameter excitations (see $5 \mathrm{a}$ ). The excitation pattern provided by ceramic is periodic along the circumference and the different combinations using 8 switches corresponding to a particular face of the blisk allows to vary the nodal diameter (ND) of the excitation among which the following particular:

- 8 groups in phase give a 0ND (mainly);

- 4 consecutive group in phase and others out of phase give 1ND excitation;

- setting successively 2 groups in phase and 2 groups out of phase gives 2 ND excitation; 


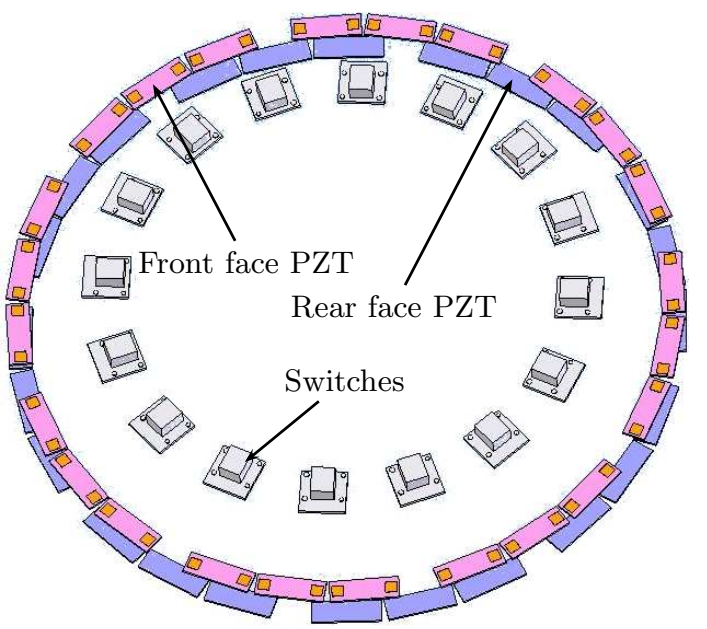

(a)

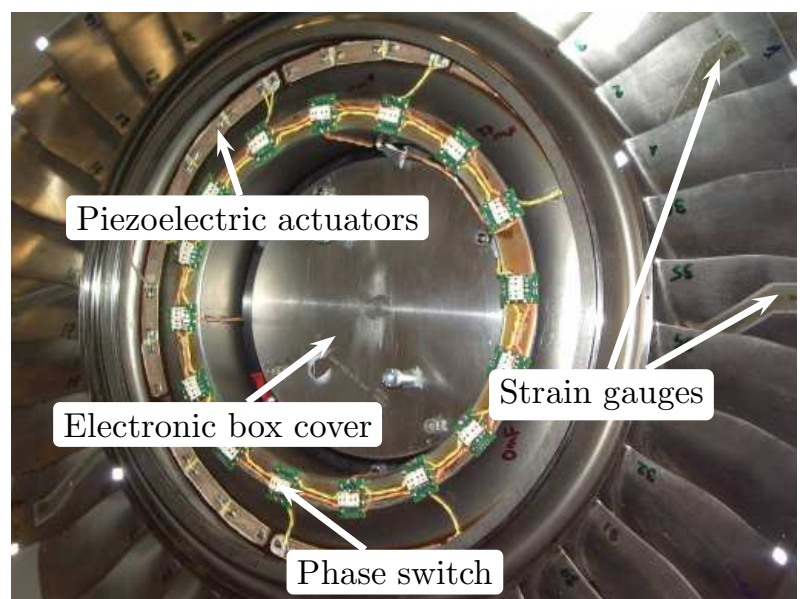

(b)

Figure 5. Details of the excitation device set up on the blisk
- setting two consecutive groups out of phase give rise to an 4 ND excitation.

These different configurations are summarised by figures 6 .

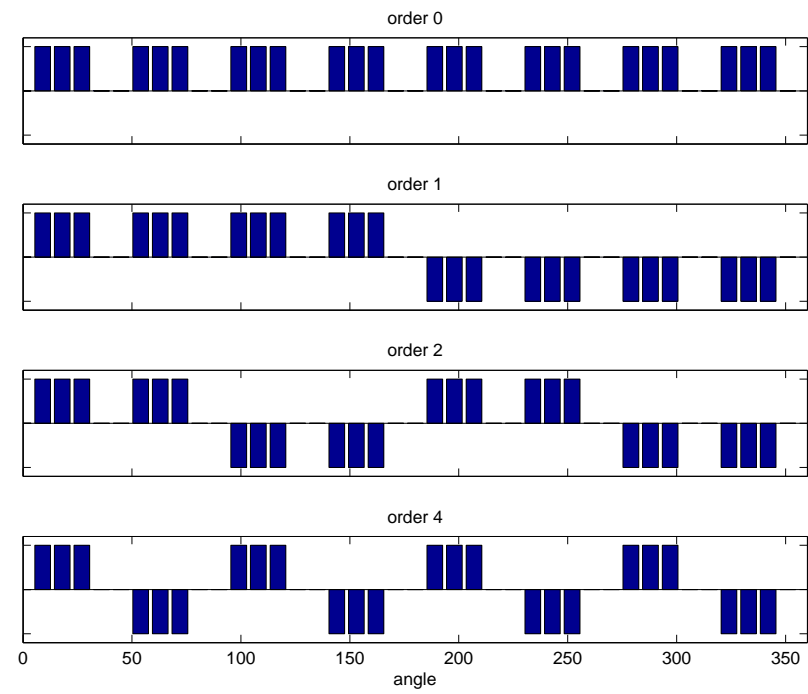

Figure 6. Settings of nodal diameters piezo-excitation.

The discussion above addresses non-rotating excitation w.r.t. the rotating frame attached to the blisk. Rotating excitations can be provided as far as the rear face of the blisk is similarly equipped with 8 groups of actuators and switches with the difference that the locations are shifted from the front face actuators with an angle of 22.5 degrees $(\pi / 8)$ along the circumference (see figure 5a). The rear and back faces PZTs are driven by two different circuits and bipolar amplifiers via an end-shaft slip-ring. The rotating excitation will be produced by means of two sine and cosine signals driving respectively the two amplifiers dedicated to each one of the disk faces.

One can note that this set-up will be optimal only for $4 \mathrm{ND}$ rotating excitations which are of main interest in this study whereas a non-resonant residual component will be obtained for 1 ND or 2 ND rotating excitations.

\subsection{Measurements}

The dynamic response of the rotating blisk is measured by means of 12 semiconductor strain gages; 8 of them are cemented on the suction side of the blades while 4 others are on the disk. The location and orientation of sensors results from a compromise 
for maximising signals that would be observed on the different target mode shapes and may be useful to easily distinguish them from one to each other especially in "veering zones". Thanks to their small size and high sensitivity, these are well suited to make measurement at high frequency with no significant perturbation of the test piece.

A telemetry system with 8 channels is used allowing signal conditioning, anti-aliasing filtering, $\mathrm{AD}$ conversion close to sensors (see figure 5), minimizing connections lengths and therefore avoiding disturbances of signals. Digital output of the 8 sensor modules are multiplexed and transmitted to the receiver in the laboratory frame using any inductive coil mounted on the rear end of the shaft. The sensor modules are assembled on a main board and the whole carefully maintained inside a metallic box fixed to the supporting disk. Switches implemented on the main board, allow selecting 8 sensors among 12 . Although voltage can also be supplied to the telemetry system by inductive coil, the slip ring is used for that purpose as it is also necessary for driving piezoelectric actuators.

Piezoelectric drivers precision voltage provide lowvoltage representations of the high-voltage output used as the reference signal for FRFs measurements. General instrumentation such as accelerometers, pressure temperature gage, tachometer is also classically used for this rig.

\subsection{Calibration of measured data}

Given the complexity of the test structure, we find appropriate to perform an experimental modal analysis of the linear (without a ring) structure at stop previous to the experiments on the non-linear system under rotation. This was done using a laser velocimetry head in combination with the strain gauges measurements. Several things can be investigated by means of such a preliminary study. First, it makes it possible to verify that the modal situation of the structure is close to what the model and the numerical analysis would predict. The modal damping and the mistuning can also be identified. Also, this study can provides useful information regarding the measured data (from strain gauges) with reference to the global mode shapes of the blisk. In particular,

- the modal participations for a given excitation configuration can be identified;

- a calibration ratio between strain gage signals and tip displacement for each mode shape can be obtained;

- for each mode, a calibration ratio between the displacement (resp. strain) on each blade and the maximum displacement (resp. strain) among all blades can be obtained.

A classical pole/residue model is used for this experimental modal identification. As an example, we provide the identified residues (denoted $R_{k}$ below) of the two 4-ND target modes in figures 7; frequency are normalized in Unit of Frequency (UF). For this test, the front side $4 \mathrm{ND}$ excitation was used. The

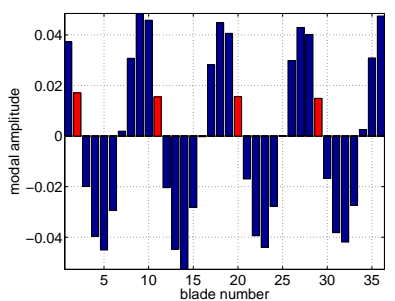

(a) 1-st mode: $111.92 \mathrm{UF}$

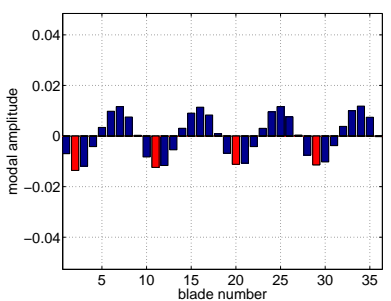

(b) 2-nd mode: $111.96 \mathrm{UF}$
Figure 7. Identified modes (4 ND); velocimetry measurements.

plots represents the identified residues of all blades obtained from the laser measurements and the red bars locate blades at which a strain gauge is present (blades \# 2, 29, 20 and 11). One can first note that the mistuning is very small for this mode but its effects (peak separation on FRFs) are still visible given the very weak damping ratios of these two modes. However, barely no localisation effect due to mistuning seems to appear and the twin modes are in spatial quadrature (cyclic symmetry).

Then, the modal participations of these two modes are clearly different for this excitation configuration as the first mode is mainly responding; the ratio of these modal participations is about 4. Also, the blade instrumented with strain gauges (in red in figures 7) correspond nearly to anti-nodes for the second mode. In summary, for the second mode, the maximum amplitude can be obtained straightforward with the signal from the instrumented blade whereas, for the first mode, a calibration ratio (about 3.1 in the mean) is to be applied on the measured signal to find the maximum modal amplitude.

Finally, the measured displacements and strain for a given blade have been compared and the ratio be- 
tween these two quantities was in accordance with the prediction from FE calculations (figure 2c).

This two ratios will be assumed independent of the non-linear effects or of the rotation effects in what follows. The validity of this assumption (not true in the general case) is checked numerically in this application.

\section{Experimental results}

In this section, the experimental results are presented. First, we propose some preliminary results in order to validate the test data. Then, parametric studies are presented in order to investigate the phenomenological aspects of friction ring damping.

\subsection{Preliminary experimental results}

In order to validate the experimental data obtained on the test rig, several preliminary tests are necessary. For example, the linearity of excitation and its efficiency are important things to verify; this was done on the blisk without friction rings. Also and as often when working with friction systems, the reproducibility of the tests has to be attested. This is particularly important when dealing with friction and contact non-linearities which are very sensitive to the loading conditions or to the environment variability in general and in particular at the contact interfaces (asperities, possible wear,... ). Several tests are needed to verify this last point among which the stability of the response (periodic regimes) or the independence of the response with respect to the test history. To verify this last point, we have considered the blisk rotating with a friction ring and we show, in figures 8, two test results in swept-sine response for various excitation levels each.

- in figure 8a, the rotation speed is $1000 \mathrm{rpm}$ and excitation level varies from $0.02 \mathrm{~V}$ to $8 \mathrm{~V}$ up and down as shown in the legend;

- figure 8b corresponds to a $2000 \mathrm{rpm}$ test and the excitation level varies from $52 \mathrm{~V}$ to $0.5 \mathrm{~V}$ down and up to $92 \mathrm{~V}$.

Comparing the first response (in solid lines) to the second ones (in dashed lines), we can notice that they are very close. In general cases, a few "up-down" tests are often required to ensure a steady -state response and, even if relative difference of about $20 \%$ (on peak

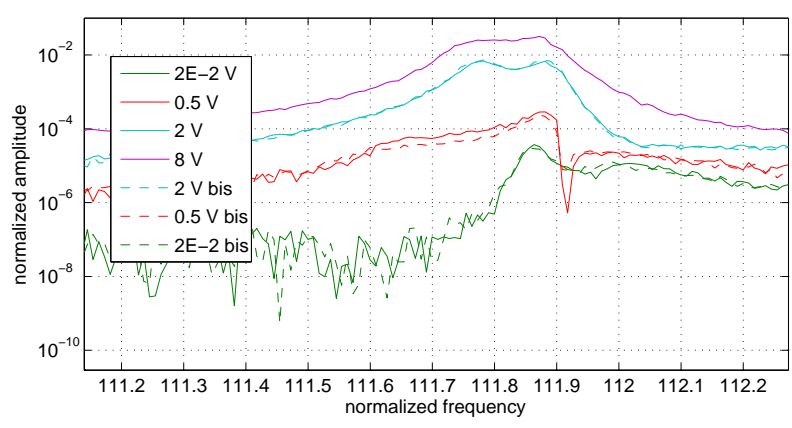

(a)

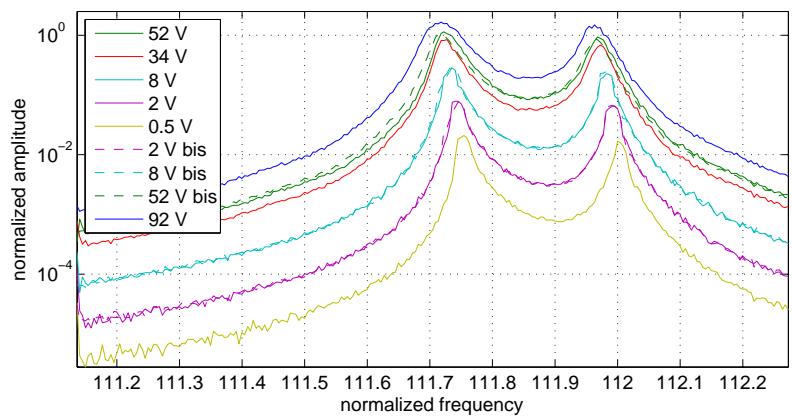

(b)

Figure 8. Reproducibility tests with the excitation level varying; (a) at $1000 \mathrm{rpm}$ and (b) $2000 \mathrm{rpm}$. 
amplitude) can be observed between two correspondent responses, the reproducibility is considered to be fair.

One can also note the effect of mistuning on these frequency response which, even if it is small, separate the resonance peaks.

\subsection{Rotating tests and parametric studies}

These preliminary verification being done, we can present the main experimental results of this study. These consists mainly of experimental parametric studies which will be used to explain some phenomenological aspects of friction ring damping. The excitation is a stepped-sine at constant level in the vicinity of the target mode's frequency. The spatial distribution is four nodal diameters on the front face of the disk. The following results corresponds to the strain measurements at blade \# 20 (see figure 7).

The first example corresponds to a $2000 \mathrm{rpm}$ test in which the excitation levels changes from 10 to 100 V. Figures 9 gather the responses (in normalized strain) along with the receptance (i.e. the response normalized by the excitation). The non-linear effects are clearly noticeable, in particular on the receptance curves. The vibration levels are about $30 \%$ less than their initial value for the maximum excitation level. We can also notice the shift of the resonance frequencies (toward lower frequencies) as the excitation level increases which is typical of frictionally damped systems.

Moreover, one can notice that the difference between the resonance frequencies has increased with respect to the previously mentioned non-rotating tests. Actually, this is due to the gyroscopic effects which are, for this mode, prominent with respect to the mistuning effects (mistuning is here very small, $0.01 \%)$.

On the second example, at $1500 \mathrm{rpm}$ (figure 10), the non-linear effects are more important. The falloff the resonance frequency is still noticeable and the vibration attenuation is more important than in the previous case.

\subsection{Efficiency of friction rings}

The previous examples illustrate the efficiency of the friction rings damping technology. A synthesis of these results is then provided in figure 11. For various tests, we have represented the evolution of the receptance (denoted $X / F$ ), for the two modes, as a

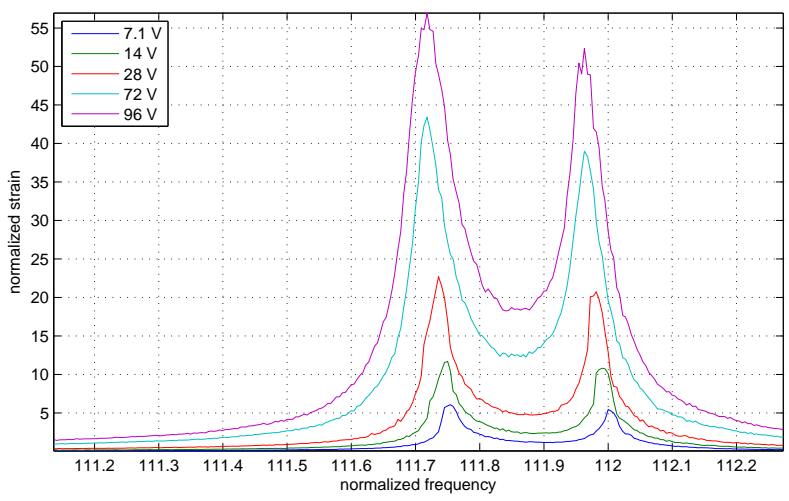

(a)

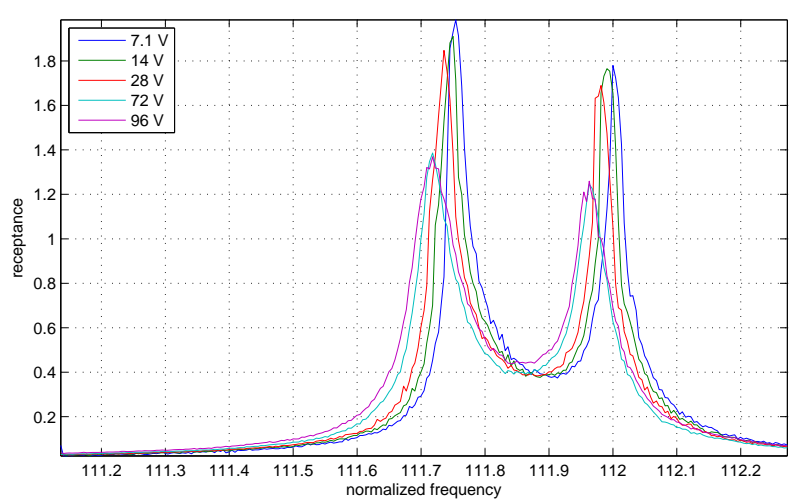

(b)

Figure 9. Experimental responses at $\Omega=2000 \mathrm{rpm}$ for various excitation levels (in Volts); (a) strain level, (b) receptance. 


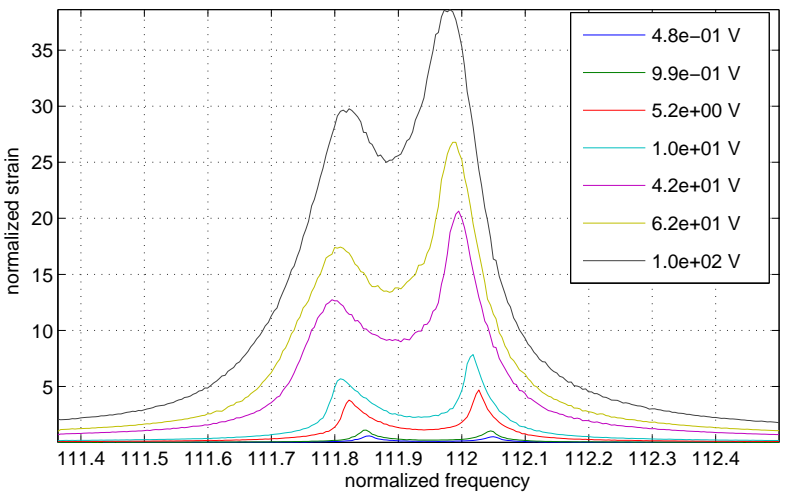

(a)

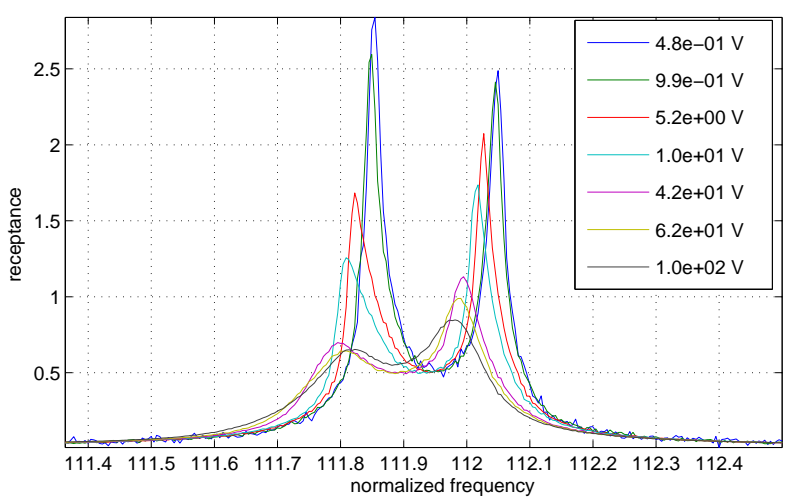

(b)

Figure 10. Experimental responses at $\Omega=1500 \mathrm{rpm}$ for various excitation levels (in Volts); (a) strain level, (b) receptance. function of the excitation level normalized by the centrifugal force $\left(N \sim \Omega^{2}\right)$. There are two data sets for

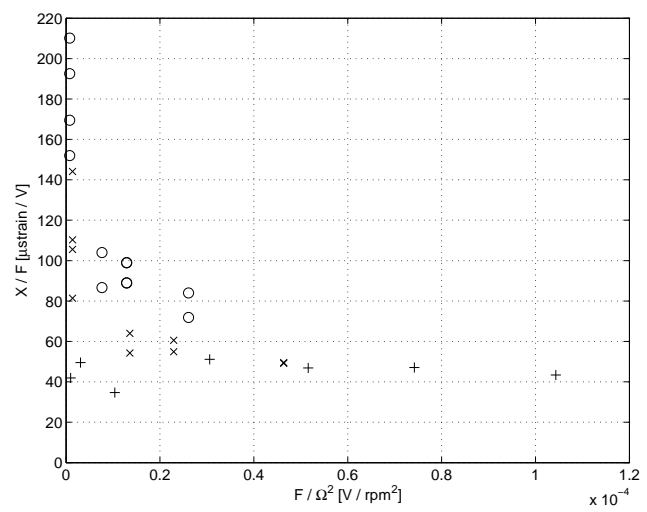

Figure 11. Diagram of efficiency of the friction rings for various rotation speed and excitation levels; (o) $2000 \mathrm{rpm}$, (×) $1500 \mathrm{rpm}$ and (+) $1000 \mathrm{rpm}$.

the two modes. We can notice a typical behaviour of frictionally damped systems since the receptance is high for low values of the ratio $F / N$ and decreases as this ratio increases. The friction damping is then optimal when the ratio of the excitation level on the normal load is large.

However, these quite simple explanation should not hide the complexity of the phenomenology of friction damping in a general point of view and particularly in this study which deals with a highly complex structure with a distributed contact interface.

\section{Identification of non-linear modal parameters}

Non-linear effects can generate significant distortions on measured FRF leading to unsatisfactory if not impossible fits when using classical linear response model based on modal analysis. As a consequence, the resulting modal parameters, namely the frequencies, damping ratios, mode shapes may be meaningless and the modal model not predictive. Based on non-linear normal modes $[9,16]$, modal analysis of experimental responses has been extended or applied to non-linear systems by several authors $[3,5,10,11]$. An extended form of the method proposed by Gibert [5] is used herein in order to take into account the use of complex modes which may result from heavily damped and closed modes together with the nonlinear effect. The method uses a modal superposition, 
although the system is obviously non-linear, but generalizes in some way the linear classical approach as shown in the following FRF model:

$h(\omega)=\sum_{k=1}^{N}\left(\frac{R_{k}\left(q_{k}\right)}{-\lambda_{k}\left(q_{k}\right)+i \omega}+\frac{R_{k}^{*}\left(q_{k}\right)}{-\lambda_{k}^{*}\left(q_{k}\right)+i \omega}\right)+E-\frac{F}{\omega^{2}}$

where

$$
\lambda_{k}\left(q_{k}\right)=-\xi_{k}\left(q_{k}\right) \cdot \omega_{k}\left(q_{k}\right)+i \omega_{k}\left(q_{k}\right) \sqrt{1-\xi_{k}^{2}\left(q_{k}\right)}
$$

and where $\xi_{k}\left(q_{k}\right), \omega_{k}\left(q_{k}\right)$ can be interpreted as nonlinear damping ratios and frequencies, depending of the modal amplitude $q_{k}$. Also, the vector $R_{k}$ can be a function of $q_{k}$. In fact, the argument of these functions is the modulus $\left|q_{k}\right|$ but we use $q_{k}$ instead in order to simplify the writing. Some classical correction terms are used by means of vectors $E, F$ in order to represent assumed non-resonant out-of-range modes participations. The size of vectors $h, R_{k}, E, F$ is the number of measuring points. Each non-linear modal participation (denoted with subscript $k$ ) are computed separately before summation in equation (1). Those coming from conjugate terms in equation (1) have been neglected here. As said before, the used experimental data are first order FRFs obtained by extracting from the quasi-steady state response the harmonic of a constant amplitude sine excitation. The frequency range can encompasses one or several non-linear resonances. The method is global as the identification process can simultaneously take into account whatever set of experimental FRFs emanating from e.g. different levels, measurement points, sweeping directions. For instance, FRF curves are sensitive to damping ratio value only near the resonance. Then, several tests performed at different levels may be needed in order to obtain a predictive modal model covering an entire amplitude range. Also, as often encountered with non-linear systems, responses presenting one or even more jumps can be handled by the present approach. Unknowns are functions $\omega_{k}, \xi_{k}, R_{k}$ of modal amplitudes $q_{k}$ which is in turn an unknown with this approach. The herein chosen representation for modal functions are polynomials of degree 3 for $\omega_{k}, \xi_{k}$ and degree 1 for $R_{k}$. Initial estimate for polynomials can result from a trial linear identification or rough peak-picking on data as explained in [5]. Then, FRFs can be iteratively synthesized according to equation (1) inside an optimisation loop, for polynomial coefficients to be determined while minimizing

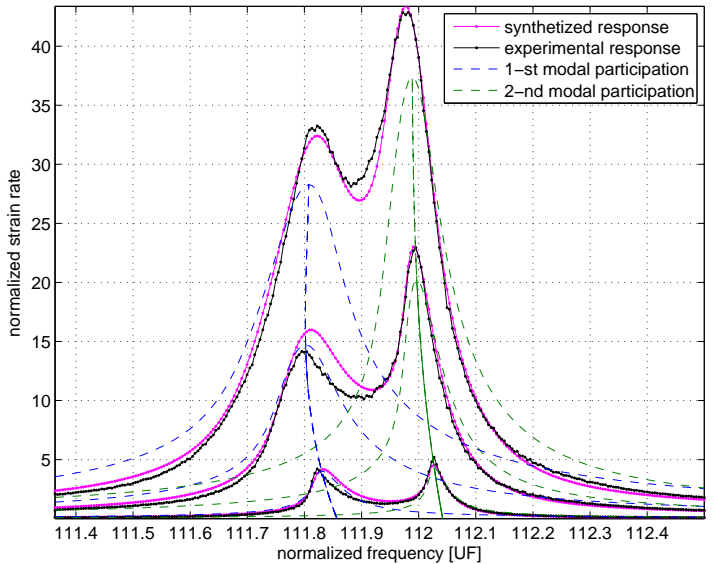

Figure 12. Example of non-linear curve fitting (blade \# 2 at $1500 \mathrm{rpm}$ )

an error function such as the basic form (3) below:

$\epsilon\left(\omega_{k}, \xi_{k}, R_{k}, E, F\right)=\sum_{i \in I} \sum_{j} p_{i}\left(\omega_{j}\right)\left|h\left(\omega_{j}\right)-F R F_{\exp }\left(\omega_{j}\right)\right|_{i}$

where $I$ stands for a set of indices for each pair of synthesized and experimental FRF to be compared and where $p_{i}$ are weighting factors. Each modal participation involved in 1 can be rapidly obtained, for a given set of parameters, by solving only a single scalar non-linear sub-problem giving bounds for $q_{k}$ and then $h(\omega)$ explicitly (see [5]). As far as input force is not directly measured in this particular application, only complex $R_{k}$ terms of 1 will be identified.

The figure 12 shows an example of curve-fitting together with two non-linear modal participations and amplitude dependant frequencies (backbone curves). The three responses are obtain at a same measurement point for several excitation levels.

The second mode gives a more important participation at that particular location given the placement of gauges discussed above. Although, as shown below, the first resonance is stronger and more linear effect is observed on that latter. This may explain why the fitting is better for the second resonance as far as "more non-linear effects" take place for the first peak resulting in more variability in data as said before.

\section{Numerical simulations and comparison with experiments}

In this section, the experimental results are compared with the ones from numerical simulations. The com- 
parison are done on the modal parameters, identified from the measurements using the previously described method and calculated using a dedicated nonlinear modal analysis method.

\subsection{Numerical methods}

In [7], a numerical method for simulations of nonlinear dynamics of systems featuring friction and contact interfaces is proposed. This approach will be briefly presented here. It is a non-linear modal analysis method which is based on a frequency domain formulation of the equations of the motion of a non-linear system along with a definition of complex non-linear modes. The concept of non-linear normal modes $[9,15,16,18]$ is extended to the case of nonlinear non-conservative systems by analogy with the linear complex modes. A complex eigenfrequency can then be defined:

$$
\lambda=-\beta+i \omega
$$

in which,

- $\omega=\omega_{0} \sqrt{1-\zeta^{2}}$ is the damped natural frequency,

- $\omega_{0}$ is the natural frequency ,

- $\zeta=\beta / \omega_{0}$ is the modal damping ratio.

We consider a discrete autonomous dynamical system governed by the following equation of the motion:

$$
\mathbf{M} \ddot{\mathbf{x}}+\mathbf{C} \dot{\mathbf{x}}+\mathbf{K x}+\mathbf{f}(\mathbf{x}, \dot{\mathbf{x}})=\mathbf{0}
$$

in which, along with typical conservative terms, potentially dissipative terms from the linear damping or from the non-linear internal forces $\mathbf{f}(\mathbf{x}, \dot{\mathbf{x}})$. The aim is to calculate the modes of this non-linear non-conservative system and as in harmonic balance methods, we postulate a form of solutions of this free vibrations problem. It takes the form a "damped" Fourier series:

$\mathbf{x}(t)=\mathbf{Q}^{0}+\sum_{n=1}^{N_{h}} e^{-n \beta t}\left(\mathbf{Q}^{n, c} \cos n \omega t+\mathbf{Q}^{n, s} \sin n \omega t\right)$,

which fundamental frequency, $\lambda=-\beta+i \omega$, is complex and represents the eigenfrequency of the mode (see Eq. (4)).

The vector gathering the Fourier cosine and sine components $\mathbf{Q}=\left\{\mathbf{Q}^{j}, j=0 \ldots N\right\}$ is the eigenvector of the complex mode.
Introducing the frequency domain differential operator:

$\nabla=\operatorname{diag}\left(0, \nabla_{1}, \ldots, \nabla_{N_{h}}\right)$ with $\nabla_{n}=n\left[\begin{array}{cc}-\beta & \omega \\ -\omega & -\beta\end{array}\right]$,

and the dynamical stiffness:

$\mathbf{Z}(\lambda)=\mathbf{K} \otimes \mathcal{I}_{2 N_{h}+1}+\mathbf{C} \otimes \mathcal{I}_{2 N_{h}+1} \nabla+\mathbf{M} \otimes \mathcal{I}_{2 N_{h}+1} \nabla^{2}$

the frequency domain eigenvalue equation can then be derived from equation (5):

$$
\mathbf{Z}(\lambda) \mathbf{Q}+\mathbf{F}(\mathbf{Q}, \lambda)=\mathbf{0}
$$

which unknowns are the eigen-parameters $\{\mathbf{Q}, \lambda\}$.

This equation is solved using an Alternating Frequency-Time method combined with a Newtonlike solver. Further details can be found in [7].

\subsection{System and contact interface modelling}

In this numerical study the blisk is represented by a cyclic symmetry FE model (see figure 2a). This model is then reduced using a Craig-Bampton approach. The rings are represented by $3 \mathrm{D}$ beam elements models. Also, mistuning is not represented in this model.

Node-to-node contact elements are introduced with both normal (contact) and tangential (2D friction) relative displacements. For the friction behaviour, we have used Dahl model [4] which is able to represent micro-slip effects in contrast with traditional "elastic slip" models (which involve the use of penalty stiffness). The restoring force for the Dahl model is commonly defined by the following implicit differential equation:

$$
\frac{d r}{d \delta}=\sigma\left(1-\frac{r}{F_{c}} \operatorname{sgn}(\dot{\delta})\right)^{\alpha}
$$

in which, $\delta$ is the tangential relative displacement of the nodes in contact, the parameters $\sigma$ and $\alpha$ define the Dahl model and $F_{c}$ is the limit Coulomb force. In particular, the parameter $\sigma$ corresponds to an equivalent tangent stiffness for small relative displacements. Examples of hysteretic cycles for a classical penalty stiffness model and a Dahl model are shown in figures 13 . These underline the influence of the microslip.

Next, the result of numerical simulations will be compared with the experimental ones. A careful 


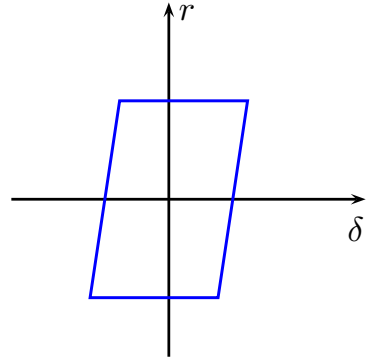

(a) Elastic slip model

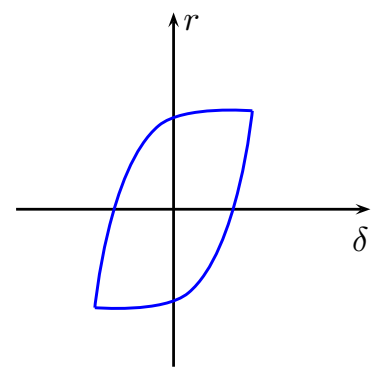

(b) Dahl model
Figure 13. Friction laws

choice of the parameters of these models has to be done previously. The first and main parameter is the coefficient of friction. As it is generally difficult to evaluate and to control, experimental studies are often needed. Moreover, the coefficient of friction features an important variability with parameters such as the temperature, the contact pressure distribution, the slip rate and velocity or, more generally, any interface characteristics (lubricant coating, wear, ... ). In this study, little information was available on this parameter and consequently, several values were considered for the comparison with the experimental results.

In addition, the Dahl model's parameters have to be defined. The parameter $\alpha$ was set to 1 , as it is commonly done and the parameter $\sigma$, which represents a stiffness for small values of the relative displacement. Several approaches exist to determine this "contact stiffness" either analytical, numerical or experimental $[13,17]$. In the present study, this was defined in relation with an elastic slip rate, $\delta_{s}$ taking into account the effects of mesh discretization by means of the mean inter-node distance $(l)$ :

$$
\sigma=\frac{F_{c}}{\delta_{s} l}
$$

In the following numerical applications, the elastic slip rate is about $10^{-5}$.

\subsection{Experimental and numerical com- parison}

The modal identification procedure presented in section 4 gives the evolution of the modal parameters (natural frequency and modal damping in particular) as a function of the vibratory amplitude whatever the shape of the excitation. The numerical methods briefly presented previously and detailed in reference [7] gives the same information (given the number of harmonics in the harmonic balance method is sufficient). Consequently, data from experimental and numerical tests are compared straightforward.

\begin{tabular}{|c|c|}
\hline $\boldsymbol{- \boldsymbol { ⿰ }}$ & 1-st mode, experimental \\
\hline $\boldsymbol{- \boldsymbol { U }}$ & 2-nd mode, experimental \\
\hline- & Dahl model, $\mu=0,3$ \\
\hline- & Dahl model, $\mu=0,5$ \\
\hline & Dahl model, $\mu=0,7$ \\
\hline
\end{tabular}

Table 1. Legend of figure 14

Figure 14 shows the comparison with the test at $1500 \mathrm{rpm}$; its legend is provided in table 1 . One
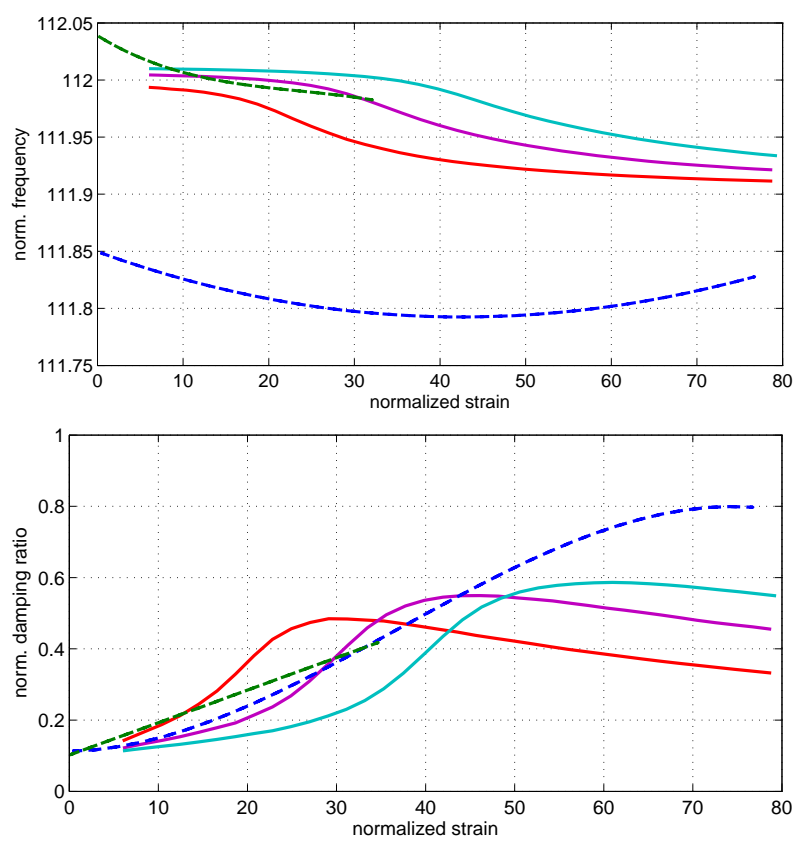

Figure 14. Experimental / numerical comparison at $1500 \mathrm{rpm}$

can first notice that the results from the numerical simulations are in the same order of magnitude as the experimental / identified data. In particular, the range of efficiency of the friction damper (in terms of vibration amplitude) as well as the level of damping are quite properly predicted by the numerical model. Next, we were more interested by the evolution of the damping ratio which is the main design parameter. The numerical simulations, whatever the coef- 
ficient of friction, lead to underestimated values of this damping ratio. Several explanations, which, at this state of the study, remain assumptions, could be proposed on this particular point:

- uncertainties and variability in the measured vibration levels;

- uncertainties on the contact conditions;

- complex friction or contact phenomena (static friction, damping due to impacts, wear,...) not taken into account in the model due to a lack of information;

- errors in the modal identification process;

- the effect of the discretization in the model;

- discrepancies on the model of the linear structure (FE model);

- the effect of mistuning.

If the initial results of this study appears encouraging, yet further works would be required both in experimental and numerical fields. From the experimental point of view, the variability of the measured data (which mainly arises from the contact conditions) need to be identified. Further investigations on the numerical aspects will mainly focus on the effects of discretization and on the contact modelling.

\section{Conclusions}

The present study focuses on the experimental characterization of friction ring damping for turbomachinery blisks and a comparison with numerical simulations. A dedicated test rig was designed at the ECL in order to study the dynamics of a real blisk under representative but controlled operating conditions. Specific excitation and measurement systems were developed in this view. The first experimental results obtained for this study and presented here show the main phenomenological aspects of this friction damping technology.

These experimental results were then compared with those from numerical simulations. We were particularly interested studying the modal parameters. The experimental ones were extracted from the experimental data by means of a non-linear modal identification technique whereas the ones from the numerical simulations were obtained through a nonlinear modal analysis technique in the frequency domain. Despite the comparisons of numerical simulations and experiments give encouraging results, additional modelling efforts seems to be necessary.

\section{Acknowledgments}

Thanks go to Snecma for its technical and financial support. This work takes place in the framework of the MAIA mechanical research and technology program sponsored by CNRS, ONERA and SAFRAN Group.

\section{References}

[1] ADTurbII. Aerolastic Design of Turbine Blades II: EU Research Project No. GRD1-1999-10258. 2000. URL: http://www.egi.kth.se/ekv/adturb2.

[2] D. Charleux, C. Gibert, F. Thouverez, and J. Dupeux. "Numerical and Experimental Study of Friction Damping in Blade Attachments of Rotating Bladed Disks". In: International Journal of Rotating Machinery (2006). Article ID 71302, 13 pages. DOI: 10.1155/I JRM/2006/71302.

[3] Y. H. Chong and M. Imregun. "Development and Application of a Nonlinear Modal Analysis Technique for MDOF Systems". In: Journal of Vibration and Control 7.2 (2001), pp. 167-179. DOI: $10.1177 / 107754630100700202$.

[4] P. R. Dahl. "Solid friction damping of mechanical vibrations". In: AIAA Journal 14.12 (1976), pp. 1675-1682.

[5] C. Gibert. "Fitting measured frequency response using non-linear modes". In: Mechanical systems and signal processing 17.1 (2003), pp. 211-218. DOI: 10.1006/mssp. 2002.1562.

[6] P. Jean, C. Gibert, C. Dupont, and J. P. Lombard. "Test-Model Correlation of Dry-Friction Damping Phenomena in Aero-Engines". In: Proceedings of ASME - Turbo Expo. GT200850891. Berlin, Germany, 2008. 
[7] D. Laxalde, L. Salles, L. Blanc, and F. Thouverez. "Non-linear modal analysis for bladed disks with friction contact interfaces". In: Proceedings of ASME Turbo Expo. Berlin, Germany, 2008, GT2007-50860. OAI: hal archives-ouvertes.fr:hal-00309682.

[8] D. Laxalde, F. Thouverez, and J.-P. Lombard. "Forced Response Analysis of Integrally Bladed Disks With Friction Ring Dampers". In: Journal of Vibration and Acoustics 132.1 (2010), p. 011013. DOI: 10.1115/1.4000763. OAI: hal.archives-ouvertes.fr:hal-00453467.

[9] R. M. Rosenberg. "The Normal Modes of Nonlinear n-degrees-of-freedom Systems". In: Journal of applied Mechanics 30 (1962), pp. 595611.

[10] S. Setio, H. D. Setio, and L. Jézéquel. "A method of non-linear modal identification from frequency response tests". In: Journal of Sound and Vibration 158.3 (1992), pp. 497-515. DOI: 10.1016/0022-460X (92) 90421-S.

[11] S. Setio, H. D. Setio, and L. Jézéquel. "Modal Analysis of Nonlinear Multi-Degree-of-Freedom Structure". In: The International Journal of Analytical and Experimental Modal Analysis 7.2 (1992), pp. 75-94.

[12] I. A. Sever, E. P. Petrov, and D. J. Ewins. "Experimental and numerical investigation of rotating bladed disk forced response using underplatform friction dampers". In: Proceedings of ASME turbo Expo. Montréal, Canada, 2007.

[13] W. Sextro. Dynamical contact problems with friction. Springer-Verlag, 2007.

[14] W. Sextro. "The Calculation of the Forced Response of Shrouded Blades with Friction Contacts and Its Experimental Verification". In: Proceedings of ASME Turbo Expo. 2000-GT540. Munich, Germany, 2000.

[15] S. W. Shaw and C. Pierre. "Normal Modes for Non-Linear Vibratory Systems". In: Journal of Sound and Vibration 164.1 (1993), pp. 85-124. DOI: $10.1006 /$ jsvi.1993.1198.

[16] W. Szemplinska-Stupnicka. "Non-linear normal modes and the generalized Ritz method in the problems of vibrations of non-linear elastic continuous systems". In: International Journal of Non-Linear Mechanics 18.2 (1983), pp. 149165. DOI: $10.1016 / 0020-7462$ (83) 90042-2.
[17] J. Szwedowicz, C. Gibert, T. P. Sommer, and R. Kellerer. "Numerical and experimental damping assessment of a thin-walled friction damper in the rotating set-up with high pressure turbine blades". In: Proceedings of ASME Turbo Expo. Barcelona, Spain, 2006.

[18] A. F. Vakakis, L. I. Manevitch, Y. V. Mikhlin, V. N. Pilipchuk, and A. A. Zevin. Normal Modes and Localization in Nonlinear Systems. Wiley series in nonlinear science, 1996. DOI: 10.1002/9783527617869. 